\title{
The continuity of the primary cosmic ray spectra measured by classical and giant EAS arrays
}

\author{
Jean-Noël Capdevielle $\dagger$ and Fabrice Cohen $\dagger \S$ \\ $\dagger$ APC, Collège de France, 11 Place Marcelin Berthelot, 75231 Paris Cedex 05, France
}

\begin{abstract}
The extrapolation of the original cosmic ray primary spectrum derived from the size spectrum measured in the Akeno classical EAS array coincides with the spectrum measured recently by the Hires Stereo experiment. After revisiting the calibrations carried in the overlapping energy region around $10^{18} \mathrm{eV}$, we discuss the consequences of the different approaches in classical arrays and giant surface arrays. The data is obtained from the size spectrum registration in the Akeno experiment with a modest space grid of 30 to $100 \mathrm{~m}$, instead of $1 \mathrm{~km}$ or more in giant arrays using density estimators in place of size and different absorption treatments for inclined cascades. While the analysis of those circumstances suggest a reduction of the energy converted from the estimators in giant surface arrays, the consistency of classical and fluorescence measurements gives more support to the GZK prediction.
\end{abstract}

$\S$ To whom correspondence should be addressed (capdev@cdf.in2p3.fr) 


\section{Introduction}

The recent comparison [1] between the differential cosmic ray spectrum measured by AGASA above $10^{18.5} \mathrm{eV}$ and the spectrum measured by HIRES indicates that quite large differences in intensities are exhibited between each measurement. It seems that larger intensities are obtained in the case of the giant surface array. The discrepancy is increasing with the primary energy. The steepening of the flux above $10^{20} \mathrm{eV}$ is not seen in AGASA contradicting with an expected GZK cut-off. In contrast, the HiRes Monocular spectrum is characterized by smaller fluxes remaining consistent with a GZK feature [2]. A comparable feature with HiRes1 has been obtained by the Hires Stereo data [3]. In spite of a lower statistics, the Stereo data is expected to give a better determination of the primary energy in reason of a more accurate estimation of the shower impact parameter than in the case of the monocular observation. In order to understand the systematic discrepancy between HiRes and AGASA (about 30\% in energy), it can be useful to examine the original energy calibration in AGASA which was used in the previous analysis in the Akeno experiment [4]. Around $10^{18} \mathrm{eV}$, the statistics of the Stereo observation has not yet become significant, however in this paper we will demonstrate that a good agreement can be realized with the original spectrum (Fig. 1) estimated from the most energetic extensive air showers recorded in the Akeno experiment.

\section{Size measurements and Primary spectrum in the Akeno experiment}

The arrangement of scintillators in original Akeno air shower experiment [5] was covered over an approximative area of $1 \mathrm{~km}^{2}$ and was characterized by a general spacing of $120 \mathrm{~m}$. The configuration of the detectors was reduced to $30 \mathrm{~m}$ in 3 regions, each of area $(90 X 90) \mathrm{m}^{2}$. The configuration of $1 \mathrm{~km}^{2}$ included a total of 156 scintillators with $1 \mathrm{~m}^{2}$ area to measure the differential size spectrum $\mathrm{J}(N)$ at $920 \mathrm{~g}-\mathrm{cm}^{-2}$ and to derive the primary flux $\mathrm{J}\left(E_{0}\right)$ up to $10^{18.8} \mathrm{eV}$. The specific lateral distribution of charged particles used in Akeno [5] for very large shower is the sum of one pair of NKG functions

$$
\rho(r)=C_{1} x^{s-2}(1+x)^{s-4.5}\left(1+C_{2} x^{d}\right)
$$

where $x=r / R_{0}, d=1.3, C_{2}=0.2, R_{0}=91.6 \mathrm{~m}$ and

$$
C_{1}=\frac{N}{2 \pi R_{0}^{2}}\left(B(s, 4.5-2 s)+C_{2} B(s+d, 4.5-d-2 s)\right)^{-1} .
$$

( $N$ is the size at ground level and $s$ is the longitudinal age parameter). This function is used to minimize the densities recorded at different distances as a function of the location of the axis and also it is used for the final integration on the distance $r$ to obtain the total size N. In the total Akeno air shower array, defined as 
Array 1 for the calibration of the giant array, the size is converted to the primary energy for $\left(10^{6} \leq N \leq 10^{9}\right)$ following:

$$
\bar{E}_{1}(e V)=3.9 \times 10^{15} \times\left(\frac{N}{10^{6}}\right)^{0.9}
$$

We have verified that this relation employed for vertical showers coincides with the results of CORSIKA[8] (version 6.16, proton primaries, QGSJET model [20]) within 2\% around $10^{18} \mathrm{eV}[9]$.

In the case of inclined showers, an average attenuation length can be expressed by $\Lambda_{e}$ $=204 \mathrm{~g}-\mathrm{cm}^{-2}$ for a zenith angle $\Theta \leq 45^{\circ}$ and the relation between inclined and vertical size can be written as:

$$
N(\Theta)=N(0) \times \exp \left(-\frac{\left(t-t_{0}\right)}{\Lambda_{e}}\right)
$$

with $t=t_{0} \sec (\Theta)$ and $t_{0}=920 \mathrm{~g}-\mathrm{cm}^{-2}$. This attenuation length is also in agreement with the longitudinal developments calculated with CORSIKA [12, 9]. After correction for the dispersion in zenith angle determination, the fluxes plotted on Fig. 1 have been obtained and expressed by a power law:

$$
J\left(E_{0}\right)=A \times\left(\frac{E_{0}}{E_{c}}\right)^{-\gamma}
$$

The parameters A and $\gamma$, as determined from Array 1 and Array 20 data[4] are tabulated on table 1

\begin{tabular}{ccccc}
\hline Array 1 & $3.4 \pm 0.3 \times 10^{-23}$ & $10^{15.7}$ & $3.02 \pm 0.03$ & $10^{15.7} \sim 10^{17.8}$ \\
Array 1 & $1.5 \pm 0.1 \times 10^{-29}$ & $10^{17.8}$ & $3.24 \pm 0.18$ & $10^{17.8} \sim 10^{19.0}$ \\
Array 20 & $1.04 \pm 0.1 \times 10^{-29}$ & $10^{17.9}$ & $3.16 \pm 0.08$ & $10^{17.9} \sim 10^{18.9}$ \\
\hline
\end{tabular}

Table 1. Best parameters in different energy regions for Array 1 and Array 20, reproduced following ref[4]. The values of the parameters are $A, E_{c}, \gamma$ are tabulated with the corresponding energy bands of validity

\section{The approach of the primary cosmic ray spectrum in giant surface arrays}

The $20 \mathrm{~km}^{2}$ array (Array 20) which was constructed before the AGASA experiment, consisted of 19 detectors (individual area of the detector $2.25 \mathrm{~m}^{2}$ ), separated by about 1 $\mathrm{km}$ from each other. The array involved 4 detectors inside the $1 \mathrm{~km}^{2}$. The registration of giant EAS with very large distances between the detectors gives statistically more chances to record low densities at large distance from the core. Furthermore, the detectors inside about 2 Moliere radii from the axis are usually saturated. The Particle Data Group (PDG) estimates that $1 \%$ only of the cascade energy lies outside a cylinder of 3.5 Moliere radii [7]. A direct access to the total size $\mathrm{N}$ from the densities becomes hopeless and a common procedure, the conversion of the density near $600 \mathrm{~m}$ from the axis, was introduced as a preliminary energy estimator [11]. 


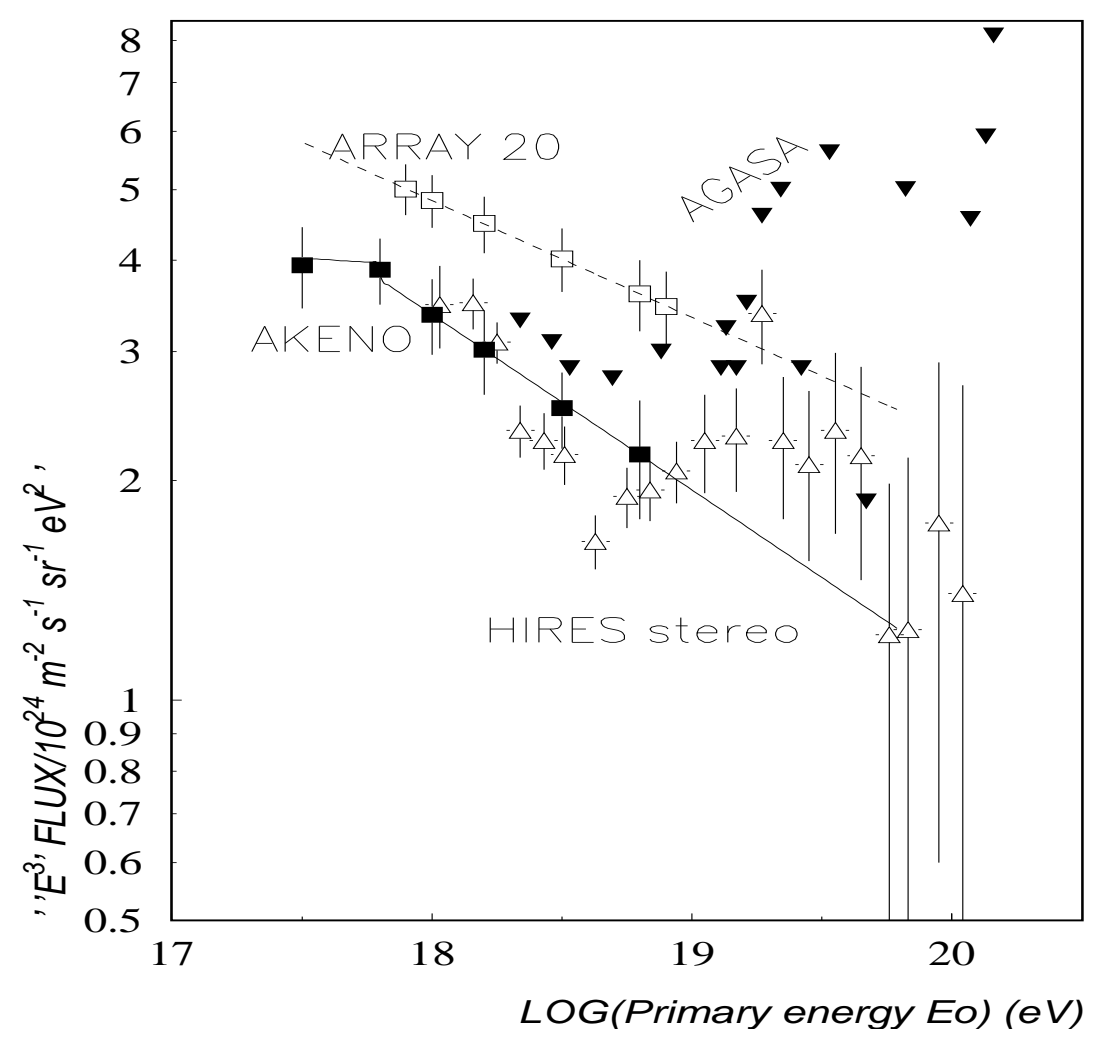

Figure 1. Differential primary spectrum for Array 1 (Akeno), Array 20, AGASA and HIRES Stereo experiments. Array 1 or Akeno (full square) concerns indifferently the data of the $1 \mathrm{~km}^{2}$ array before 1992. Array 20 (open square) concerns the earliest data of the $20 \mathrm{~km}^{2}$ array . AGASA (full triangle) is the data of the $100 \mathrm{~km}^{2}$ array in 2003 and Hires Stereo labels the Stereo data in 2003 . The fitted spectra, for Akeno (full line) and Array 20 (dashed line) corresponds to Table 1. : for the clarity of the graph, the error bars are not plotted for AGASA data.

In such context, the lateral distribution for the Array 20 has been selected as follows:

$$
\rho(r)=N C_{e} x^{-\alpha}(1+x)^{-(\eta-\alpha)}\left(1+\frac{r}{2000}\right)^{-0.5}
$$

$\left(C_{e}\right.$ being a normalization constant) This analytic description with a fixed value $\alpha=$ 1.2 , without reference to the age parameter is used to determine the shower axis and to interpolate the value of the density at $600 \mathrm{~m}$. In contrast to the size conversion in Array 1 , the scintillator response in terms of density $\mathrm{S}_{600}$ is here converted to the primary energy following:

$$
\overline{E_{20}}(e V)=2.0 \times 10^{17} \times\left(S_{600}\right)^{1.0}
$$

This energy estimation takes into account the relation between $\mathrm{S}_{600}$ and $\rho(600)$ following calibrations with the arrays of Haverah Park and Yakutsk [14]. 
The quantity S(r) can be related to the electron and muon densities [13] following:

$$
S(r)=\frac{\left(\rho_{e}(r)+\rho_{\mu}(r) k_{\mu}(r)\right)}{k_{\text {array }}} \times k_{s c}(r)
$$

with $k_{\mu}(r)=1.8\left(E_{\mu} \geq 1 G e V\right), k_{s c}(r)=1.4($ for $r=600 \mathrm{~m})$ and $k_{\text {array }}=1.1$ The original values were obtained in Akeno by comparing the acceptance of Array 20 to Array 1 as $k_{\text {array }}=1.1$ and from comparison of scintillator densities to spark chamber densities as $k_{s c}(r)=1.1$. This last value was underestimated at large distances and a value of 1.4 has to be taken at $600 \mathrm{~m}$ from the axis [13] From a set of 40 vertical showers simulated with CORSIKA (proton primaries, GHEISHA option) at $10^{18} \mathrm{eV}$, we obtain at $600 \mathrm{~m}$ the average electron and muon densities of 3.3 and 1.2 respectively. The value $k_{\mu}(r)=1.8$ has been obtained from the muons densities recorded for $\left(E_{\mu} \geq 1 \mathrm{GeV}\right.$ at $600 \mathrm{~m}$ distance by the muon detectors contained in Array 20. Taking into account the energy thresholds for electrons and muons in CORSIKA ( 1.5 and $300 \mathrm{MeV}$ respectively), the conversion of $\mathrm{S}_{600}$ in Array 20 (a value $k_{\mu}(600)=1.4$ has been assumed according to the muon energy threshold selected in CORSIKA instead of $1 \mathrm{GeV}$, taking the muon energy spectrum at $600 \mathrm{~m}$ [9]) appears to overestimate the primary energy by about $20 \%$, even if we select the FLUKA or UrQMD options of CORSIKA [9, 10], which are more favourable than the GHEISHA option . The average electron and muon densities from CORSIKA return here via relation $(7) E_{0}=1.26 \times 10^{18} \mathrm{eV}$, respectively $1.19 \times 10^{18}$ $\mathrm{eV}$ for Fluka, instead of the primary energy $E_{0}=10^{18} \mathrm{eV}$ set in our simulation. In other words, we received from our simulation (CORSIKA, option UrQMD)an average density $\mathrm{S}_{600}=6.0$ from equation(8) involving the average electron and muon densities calculated, when $\mathrm{S}_{600}=5.0$ was expected according to the conversion of Array 20 ; this minimal overestimation of the primary energy by about $20 \%$ remains approximately constant up to $10^{20} \mathrm{eV}$ in reason of the quasi-linear dependence of $\mathrm{E}_{0}$ on $\mathrm{S}_{600}$.

\section{The attenuation length for density estimators}

In place of the size spectrum, the $S_{600}$ differential spectrum in Array 20 is obtained taking an attenuation length $\Lambda_{600}$ in parallel to $\Lambda_{e}$ in Array 1 following:

$$
S_{600}(\Theta)=S_{600}(0) \times \exp \left(-\frac{\left(t-t_{0}\right)}{\Lambda_{600}}\right)
$$

A constant value $\Lambda_{600}=500 \mathrm{~g}-\mathrm{cm}^{-2}$ was assumed according to the best fit value on the zenith angle distribution for constant $S_{600}$ adjusted by the simulations [14] for different $\Lambda_{600}$ This conversion is also valid in AGASA for $\Theta \leq 45^{\circ}$.

The intensity exceeds by a factor 1.5 the primary spectrum obtained with Array 20 in the overlapping region with Akeno (the corresponding points are plotted on fig. 1 , together with the best fitt described in Table 1 and reproduced following the best adjustment [4]) was immediately explained by the different methods used for the energy reconstruction in each array. A discrepancy by a factor 1.15 in the primary energy 
derived from equation (7) instead of equation (3) was pointed out and considered as in agreement with the energy determination via $S_{600}$ in the experiments of Haverah Park and Yakutsk. Those ambiguities have been treated later [16] in terms of systematic errors on detectors response versus zenith angle, seasonal variance and other complex problems related to the shower selection and the collecting area.

The most recent values reported by AGASA [17] are more close from the values of Akeno than the values of Array 20 (figure 1) ; the intensities of AGASA remain however larger than for Array 1 in the overlapping energy region and exhibit a general excess by $30 \%$ when compared to Hires Stereo data. From our simulation data, we have derived the values of the attenuation length $\Lambda_{600}$ for different zenith angles (fig.2); for small inclinations $\Theta \leq 30^{\circ}$ the values of the attenuation length concerning proton primaries are quite more important than the average value $\Lambda_{600}=500 \mathrm{~g}-\mathrm{cm}^{-2}$ used in AGASA. When the primary energy is increasing, the depth of the maximum becomes more and more close of the arrays in altitude, such as AUGER or AGASA : the conversion of inclined densities to $S_{600}(0)$ according to equation (9) becomes poorly appropriate as the cascade is far from a stable absorption phase, especially for protons primaries. In the depth interval of about 5 radiation units following the maximum, we can summarize the absorption process as follows:

- the total size $\mathrm{N}$ is decrasing slowly versus the atmospheric depth $\mathrm{t}$

- the age parameter increases in parallel from 1.0 up to 1.2

- the lateral distribution around $600 \mathrm{~m}$ from the axis becomes flatter [9]

The increase of the flattening of the density distribution turns to a systematic overestimation (via relation (9)) of the vertical density which is reported on the primary energy and the shower recorded may be classified in bins of larger energy. The behaviour of $\Lambda_{600}$ on fig.2 at $10^{18} \mathrm{eV}$ will be emphasized at $10^{20} \mathrm{eV}$ as suggested by the values plotted for electrons only. The overestimation on the primary energy from the densities converted by formula (9), using $500 \mathrm{~g}-\mathrm{cm}^{-2}$ instead of $2000 \mathrm{~g}-\mathrm{cm}^{-2}$ in the overlapping region for $\Theta=20^{\circ}$ is about $10 \%$. The adaptation of the conversion of the densities of inclined showers, the ambiguities on the scintillator response and the relation (7) can probably explain the discrepancies of $30 \%$ up to $2.10^{19} \mathrm{eV}$. It must be observed that the estimations of $\Lambda_{600}$ are performed with pairs of set of 40 showers ; such statistics limit the convergence and the accuracy of the determination of the attenuation length is about $15 \%$

Above $3.510^{19} \mathrm{eV}$ a clear divergence in the discrepancies between AGASA and Hires Stereo appears rising from $150 \%$ above $300 \%$ at $6.10^{19} \mathrm{eV}$. This may come again from the lateral distribution becoming flatter more rapidly than the reduction of the total size : the net result is that the densities (at $600 \mathrm{~m}$ ) are $5-10 \%$ larger in the bin $\Theta=20^{\circ}-30^{\circ}$ than the vertical density when the atmospheric depth separating the array and the shower maximum becomes lower than 3 cascade units. Some systematic errors could also enter in the position of the shower axis [15] as the relation (6) is constant in the central part ( $\alpha$ is fixed to 1.2), with consequences on the estimator $S_{600}$. 


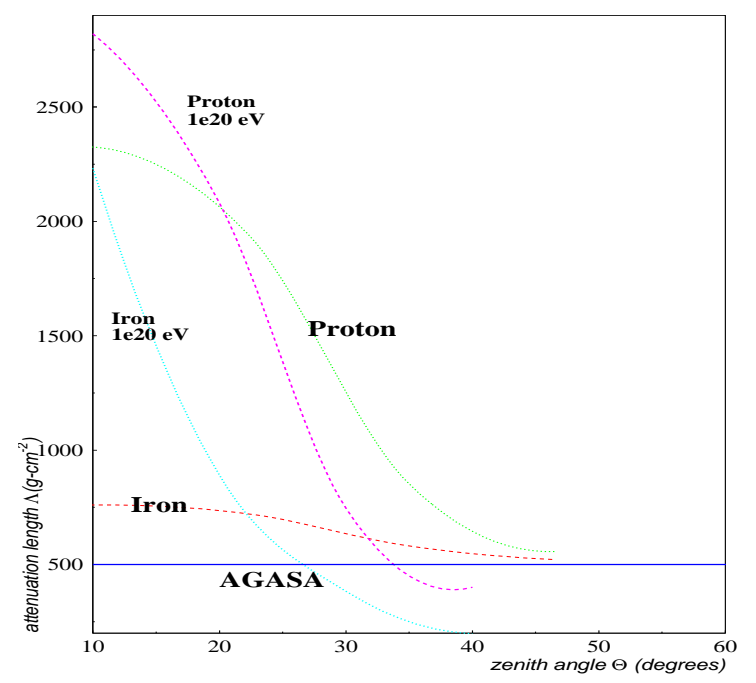

Figure 2. Attenuation length of $S_{600}$ for $\theta=10^{\circ}-50^{\circ}$ compared to the earliest assumption of AGASA in the case of proton and iron primaries, labelled respectively PROTON and IRON at $10^{18} \mathrm{eV}$. The behavior of the attenuation length at $10^{20} \mathrm{eV}$ restricted to the electrons is superimposed.

To illustrate the complex behavior of the estimator versus zenith angle in the neighbourhood of the maximum, we have reported on table 2 the relative dependence on zenith angle at $10^{20} \mathrm{eV}$ for $s(\Theta)=S_{600}(\Theta) / S_{600}(0)$ and $\delta_{v m e}(\Theta)=\Delta_{v m e}\left(\Theta / \Delta_{v m e}(0)\right.$ in the case of water Cerenkov tanks, like in Haverah Park or AUGER, for vertical muon equivalents $\left(\Delta_{v m e}(\Theta)\right.$ is the average density of vertical muons equivalent recorded at the distance considered, here $600 \mathrm{~m}$ ). This data has been obtained in a common situation at a vertical depth of $1.2 X_{0}$ under the maximum, $X_{0}$ being the electron radiation length for proton primaries (respectively2.5 $X_{0}$ for iron primaries) and allows a comparison at AUGER level $\left(870 \mathrm{~g}-\mathrm{cm}^{-2}\right)$.

Table 2. Relative dependence of estimators at $600 \mathrm{~m}$ on zenith angle for proton and iron primaries at $10^{20} \mathrm{eV}$. The ratio to the vertical densities are tabulated for scintillators $(s(\Theta))$ and water Cerenkov detectors $\left(\delta_{v m e}(\Theta)\right)$

\begin{tabular}{ccccccc}
\hline$\Theta$ & $0^{\circ}$ & $10^{\circ}$ & $20^{\circ}$ & $30^{\circ}$ & $40^{\circ}$ & $50^{\circ}$ \\
\hline$s(\Theta), \mathrm{p}$ & 1. & 1.05 & 1.08 & 1.17 & 1.0 & 0.65 \\
$s(\Theta), \mathrm{Fe}$ & 1. & 1.01 & 1.01 & 0.94 & 0.80 & 0.47 \\
$\delta_{\text {vme }}(\Theta), \mathrm{p}$ & 1. & 1.06 & 1.09 & 1.13 & 1.02 & 0.70 \\
$\delta_{\text {vme }}(\Theta), \mathrm{Fe}$ & 1. & 1.01 & 1.01 & 0.97 & 0.83 & 0.53 \\
\hline
\end{tabular}

Being based on our Monte Carlo calculation, we ascertain a maximal increase of the densities near $30^{\circ}$ for the protons by $17 \%$ for scintillators and by $12.6 \%$ for water 
Cerenkov tanks (those proportions are average quantities on groups of 40 showers). This can be the origin of an overestimation of the primary energy in comparable proportions.

For iron primaries, the situation is more stable but the average excess in vertical density, $S_{600}(0)$ or $\Delta_{v m e}(0)$, is respectively $26 \%$ and $30 \%$ against protons: this discrepancy decreases when $\Theta$ increases with similar values of the estimators at $30^{\circ}$ for scintillators and at $45^{\circ}$ for the water Cerenkov tanks. The conversion to the primary energy for scintillators is then comparable for protons and heavy primaries only near $30^{\circ}$; the relation $(9)$ provides an inappropriate description for the absorption generating an energy overestimation for protons in the band $10^{\circ}-40^{\circ}$ and a constant overestimation up to $30^{\circ}$ )for iron primaries.

On the contrary the error on the localizations of the estimators at $800 \mathrm{~m}$ or $1000 \mathrm{~m}$ do not change the situation for a heavy primary component $; s(0)$ and $\delta_{v m e}(0)$ are increasing similarly, by $26 \%$ and $30 \%$ respectively at each distance, when passing from proton to iron (Those values are obtained from the respective densities at axis distances of $800 \mathrm{~m}$ and $1000 \mathrm{~m})$. Furthermore, for iron, s and $\delta_{v m e}$ do not depend on $\Theta$ up to $30^{\circ}$.

In the case of protons, the maximal enhancement near $30^{\circ}$ appears reduced at $800 \mathrm{~m}$ from the axis ( $11 \%$ for both s and $\delta_{v m e}$ instead of $26 \%$ at $1000 \mathrm{~m}$ ). For giant showers and detectors separation by $1000 \mathrm{~m}$ or more, the accuracy on the density interpolation might be improved (a larger number of detectors hit are located at distances lower than $800 \mathrm{~m}$ ) and there could be some advantages to move the estimator at $800 \mathrm{~m}$.

\section{Conclusion}

An impressive data of high quality has been collected in AGASA. Further simulations with CORSIKA, even with fastened versions (hybrid Monte carlo and anlytic codes) to estimate more carefully the array response with a huge statistics, completed by simulations with GEANT for the scintillator response and carried in close contact with the experience, may help to clarify in detail the discrepancies between the Surface arrays and the fluorescence observatories. The present approach points out a better consistence between the spectra obtained by classical size measurements and Hires Stereo measurements. This tendency favours the GZK prediction. The spectrum measured by the array KASCADE-Grande will be useful to improve the calibration of giant surface arrays [19].

We observe on Fig. 1 that the singularity in the differential spectrum, ascertained 15 years ago in Haverah Park, Akeno, AGASA and Yakutsk [18] appears confirmed here for the different experiments with a "dip" at $4-7.10^{18} \mathrm{eV}$; it could indicate the end of the galactic component, relieved by the contribution of an extragalactic component, or the accumulation of the photonic showers coming fom the most energetic protons interacting with the black body radiation[21]. We note also that a heavy composition will reduce the intensities of AGASA, but will still be in conflict with the fluctuations of $T_{\max }$ measured with the Fly's Eye [22, 23]. 
[1] A.V. Olinto, Proc. $28^{\text {th }}$ ICRC,Tsukuba, 8, 299 (2003)

[2] D. Bergman et al., Hires Collaboration,Proc. $28^{\text {th }}$ ICRC,Tsukuba, 1, 299 (2003)

[3] R. W. Springer et al, Hires Collaboration,Proc. $28^{\text {th }}$ ICRC,Tsukuba, 1, 413 (2003)

[4] M. Nagano et al., J.Phys.G, Nucl.Part.Phys., 18, 423 (1992)

[5] M. Nagano and al, Journal Phys. Soc. Japan, 53, 1667 (1984).

[6] J.N. Capdevielle, F. Cohen and K. Sanosyan, Proc.28 ${ }^{\text {th }}$ ICRC, Tsukuba, 3, 1623 (2003).

[7] P. D. G., Phys. Rev. D 54, 137 (1996).

[8] D. Heck, J. Knapp, J. N. Capdevielle, G. Schatz and T. Thouw FZK A report-6019 ed. FZK. The CORSIKA Air Shower Simulation Program, Karlsruhe (1998).

[9] J. N. Capdevielle and F. Cohen, J.Phys. G, Nucl. Part. Phys.,31, 507-524 (2005)

[10] H.J. Drescher, G. Farrar, M. Bleicher, M. Reiter, S. Soff and H. Stcker, Proc. $28^{t h}$ ICRC, Tsukuba, 2, 507 (2003).

[11] A. M. Hillas, D.J. Marsden, J.D. Hollows and H.W. Hunter, Proc.12 ${ }^{\text {th }}$ ICRC,Hobart, 3, 1001 (1971).

[12] F. Cohen, Ph. D. dissertation, Univ. Paris XI (2003).

[13] A. Lagutin, R.I. Raikin, N. Inoue and A. Misaki, J. Phys. G, 28, 1259 (2002).

[14] H. Y. Dai, K. Kasahara, Y. Matsubara, M. Nagano and M. Teshima, J. Phys. G, Nucl. Phys., 14 793, 805 (1988)

[15] J. N. Capdevielle, C. Le Gall, J. Gawin, I. Kurp, B. Szabelska, J. Szabelski and T. Wibig, Nuovo Cimento 25C 393, 424 (2002).

[16] S. Yoshida et al., Astrop. Phys. 3, 105 (1995).

[17] M. Takeda et al., (AGASA collaboration), $28^{\text {th }}$ ICRC,Tsukuba, 1, 381 (2003)

[18] A. A. Watson, "Astrophysical Aspects of the most energetic cosmic rays", 2, ed.M.Nagano, F. Takahara, World Scientific (1990)

[19] C. Buttner et al., Proc. $28^{\text {th }}$ ICRC,Tsukuba, 1,33 (2003)

[20] N. N. Kalmykov, S. S. Ostapchenko and A. I. Pavlov, Nuclear Physics B 52B, 17 (1997).

[21] J. Lloyd-Evans, Proc. 22 ${ }^{\text {nd }}$ ICRC, Dublin,5, 226-240 (1991)

[22] P. Sokolsky, P. Sommers and B. Dawson, Physics Reports, 217, 5, 253-277 (1992)

[23] J. N. Capdevielle and R. Attallah, J.Phys. G., Nucl. and Part. Phys., 21, 121-127 (1995) 